\title{
Papers
}

\section{Stillbirth as risk factor for depression and anxiety in the subsequent pregnancy: cohort study}

\author{
P M Hughes, P Turton, C D H Evans
}

\begin{abstract}
Objective To assess women's symptoms of depression and anxiety during pregnancy and the postpartum year in the pregnancy after stillbirth; to assess relevance of time since loss.

Design Cohort study with four assessments: in third trimester and 6 weeks, 6 months, and 12 months after birth.

Setting Outpatient departments of three district general hospitals; subjects' homes.

Subjects 60 women whose previous pregnancy ended in stillbirth after 18 weeks' gestation; 60 matched controls.

Main outcome measures Depression and anxiety measured by Edinburgh postnatal depression scale, Beck depression inventory, and Spielberger state-trait anxiety scale.
\end{abstract}

Results In the third trimester women whose previous pregnancy had ended in stillbirth were significantly more depressed than control women (10.8 v 8.2; $\mathrm{P}=0.004)$ and had greater state anxiety (39.8 $v 32.8$, $\mathrm{P}=0.003)$ The difference was accounted for by those women who conceived less than 12 months after the stillbirth, who were also more depressed at 1 year. Results in those who conceived 12 months or more after stillbirth were similar to those in their controls at all points and showed lower trait anxiety 1 year post partum. One year after the birth $8 \%$ of control women and $19 \%$ of subjects scored high for depression $(\mathrm{P}=0.39)$, with most of the depression among the more recently bereaved $(28 \% v 11 \% ; \mathrm{P}=0.18)$. In the women who had experienced stillbirth, depression in the third trimester was highly predictive of depression 1 year after subsequent birth $(\mathrm{P} \leqslant 0.0005)$.

Conclusion Vulnerability to depression and anxiety in the next pregnancy and puerperium is related to time since stillbirth, with more recently bereaved women at significantly greater risk than controls. As there are problems for mother and infant associated with high anxiety and depression during and after pregnancy, there may be advantage in waiting 12 months before the next conception.

\section{Introduction}

In England and Wales 0.5\% of pregnancies end in stillbirth after 24 weeks' gestation. ${ }^{1}$ Parents inevitably suffer a process of grief and mourning which may last a year or more. ${ }^{2}$ There has been debate about when women should conceive again, with some clinicians arguing that they need time to recover emotionally before embarking on another pregnancy ${ }^{3}$ but with little systematic evidence to support this view. Studies consistently report that about $50 \%$ of women become pregnant within 12 months after loss. ${ }^{2}$ There are descriptive reports of the psychological difficulties of pregnancy after stillbirth, ${ }^{56}$ but the limited research is from small numbers ${ }^{7}$ or specialised groups. ${ }^{8}$ Recent findings that maternal anxiety and depression during and after pregnancy have deleterious effects on mother and infant reinforce the clinical relevance of the issue.

Maternal anxiety in pregnancy is associated with earlier births and lower birthweights ${ }^{910}$ and impairment of fetal brain development. ${ }^{11}$ Mediating mechanisms may include abnormal uterine blood flow ${ }^{12}$ and increased cortisol transfer from mother to fetus. ${ }^{13}$ Antenatal depression is associated with poor clinic attendance and poor self care ${ }^{14}$ and postnatal depression is associated with infant cognitive delay and emotional problems ${ }^{15}$ which may have long term effects. ${ }^{16}$

We followed 60 pregnant women who had had stillbirth or late miscarriage and 60 matched control women and evaluated depression and anxiety and the relation between time since loss and symptom levels. There were four assessments between the third trimester and 12 months post partum.

\section{Participants and methods}

\section{Participants}

Criteria for inclusion were women whose previous pregnancy ended in loss after 18 weeks' gestation, had no live children, were aged over 20 years, had a partner, had a singleton pregnancy, spoke English, and were progressing satisfactorily in the present pregnancy. We excluded women receiving treatment for acute physical or mental illness and those with previous termination for abnormality.

The 60 control women were primigravida but otherwise met above criteria and were case matched for age, socioeconomic group, and ethnic origin.

\section{Instruments}

We used four measures to assess depression and anxiety: an observer rated demographic questionnaire; the Edinburgh postnatal depression scale (a 10 item self report scale developed for postnatal use ${ }^{17}$ and now

\author{
Department of \\ Psychiatry, \\ St George's \\ Hospital Medical \\ School, London \\ SW17 ORE \\ P M Hughes, \\ senior lecturer \\ P Turton, \\ research fellow \\ Tavistock and \\ Portman NHS \\ Trust, Tavistock \\ Clinic, London \\ NW3 5BA \\ C D H Evans, \\ associate research and \\ development director \\ Correspondence to: \\ Dr Hughes \\ p.hughes@ \\ sghms.ac.uk
} BMJ 1999;318:1721-4 
validated for use during pregnancy ${ }^{18}$; for dichotomous analysis the 14/15 cut off was used antenatally (as recommended by Murray and Cox because of the high levels of dysphoria in pregnancy ${ }^{18}$ ) and for measurement at 6 weeks the 12/13 cut off was used); the Beck depression inventory (10 item self report questionnaire) ${ }^{19}$; and the Spielberger state-trait inventory (a 40 item questionnaire measuring anxiety at time of testing (state) and general tendency to anxiety (trait)). ${ }^{20}$

\section{Procedure}

About 30000 sets of case notes were screened at three district general hospitals. Controls were simultaneously identified in the same antenatal clinics by using casenotes to find primigravida matched on ethnicity, age, and socioeconomic status. A letter was sent to all identified women in the third trimester of the current pregnancy inviting them to take part. Four assessments were done: during the third trimester and at 6 weeks, 26 weeks, and 12 months after the birth. Assessment included a demographic questionnaire, Edinburgh postnatal depression scale (antenatal, 6 weeks), Beck depression inventory (6, 12 months), and Spielberger state-trait inventory (third trimester, 12 months). Interviews were carried out at the participant's home or in the outpatient departments. The procedure had approval from all local ethics committees.

\section{Statistical analyses}

Groups were compared at the four time points by using paired tests on all measures. Data for depression scores were analysed with both continuous scores and dichotomous scores with a cut off 14/15 (antenatal) and 12/13 (6 weeks postnatal) for the Edinburgh scale and 10/11 for Beck's inventory. Continuous data deviated from Gaussian distribution in one group or the other on skewness, kurtosis, or Komolgorov-Smirnov test for all variables except Spielberger trait anxiety at baseline. Hence non-parametric Wilcoxon tests are reported for paired comparisons. All analyses are reported for pairs with complete data on the dependent variable, hence $\mathrm{n}$ varies. Analyses to compare time since loss were conducted for gaps of 9, 12, 15, and 18 months between loss and conception. Parametric 95\% confidence intervals are reported for all comparisons. Comparisons of prevalences for dichotomised variables are expressed as $95 \%$ confidence intervals for relative risk.

\section{Results}

\section{Participants included and lost}

Of 96 subjects who met the inclusion criteria, 82 (86\%) agreed to participate. Thirteen (14\%) delivered before the first interview, and $69(72 \%)$ had a third trimester interview. Four were excluded after the birth because of illness or injury; data from five who were interviewed were unmatched or very incomplete. Seven dropped out later (three moved abroad, four refused to continue).

Of 83 controls approached, $63(76 \%)$ agreed to participate. One delivered before the interview; 62 $(75 \%)$ had a third trimester interview. One was excluded because of illness, and seven dropped out (two moved abroad, five refused to continue). Sixty matched pairs had a first interview; 53 pairs completed to 1 year post partum.

\section{Characteristics of sample}

The mean (range) age of participants was 30 (20-46) years for women who had experienced stillbirth and 29 (20-43) years for control women. There were 39 pairs of white women, seven pairs of Afro-Caribbean women, nine pairs of Indian or Pakistani women, four pairs of African women, and one pair of Chinese women. There was no significant difference between groups on educational level or material situation. Four subjects and two control women reported previous physical illness and four subjects and six control women previous mental illness; none had been admitted to hospital. No women reported that they had received psychiatric treatment after the stillbirth. Six women reported that the stillborn infant had a congenital abnormality; the remainder had been told the infant was normal. Ten subjects who had undergone previous termination of pregnancy showed no significant differences from other subjects on baseline variables. Thirty one women (52\%) conceived less than 12 (range 2-11; median 5) months after loss and $29(48 \%)$ more than $12(12-180 ; 23)$ months after loss.

\section{Results}

Third trimester

Stillbirth and control groups differed significantly in depression scores (mean for stillbirth group $10.8 v 8.2$ in control group; Wilcoxon test $\mathrm{P}=0.004 ; 95 \%$ confidence interval for difference 0.7 to 4.5 ). With $14 / 15$ cut off for the Edinburgh scale ${ }^{18} 17(28 \%)$ of the stillbirth group and $5(8 \%)$ of the control group scored high (McNemar $\mathrm{P}=0.01 ; 95 \%$ confidence interval for relative risk 1.3 to $16)$, with a significant difference on state anxiety (39.8 $v$ 32.8; Wilcoxon $\mathrm{P}=0.003$; $95 \%$ confidence interval for difference 2.6 to 11.2 ) but not trait anxiety (38.4 v 35.8; Wilcoxon $\mathrm{P}=0.11 ;-1.1$ to 6.3 ).

\section{Further assessments}

At 6 weeks there was no significant difference between groups on Edinburgh depression scores (7.3 v 7.0; Wilcoxon $\mathrm{P}=0.72 ;-1.4$ to 1.9 ). With $12 / 13$ cut off, six $(10 \%)$ in the stillbirth group and three $(5 \%)$ in the control group scored high (McNemar $\mathrm{P}=0.51 ; 95 \%$ confidence interval for relative risk 0.431 to 12.4). At 26 weeks there was no significant difference between groups on depression scores (Beck $5.9 v 6.3$; Wilcoxon $\mathrm{P}=0.59 ; 95 \%$ confidence interval for difference -2.8 to 2.0$)$. With $10 / 11$ cut off, four $(7 \%)$ in the stillbirth group and seven (12\%) in the control group scored high (McNemar $\mathrm{P}=0.32 ; 95 \%$ confidence interval for relative risk 0.4 to 12.4). There was no significant difference between anxiety and depressive symptoms at 1 year (Beck 6.0 v 5.1; Wilcoxon $\mathrm{P}=0.44$; $95 \%$ confidence interval for difference -1.1 to 2.9 ; Spielberger state 31.7 v $32.5 ; \mathrm{P}=0.57 ;-5.1$ to 3.6 ; trait 34.8 v 36.4 ; $\mathrm{P}=0.46 ;-6.0$ to 2.7$)$. Dichotomy of the Beck score at $10 / 11$ showed a trend towards higher symptoms in the index group (11 (19\%) v 5 (8\%); McNemar $\mathrm{P}=0.39$; $95 \%$ confidence interval for relative risk 0.1 to 1.9 ).

Relation between time from loss to conception and symptom levels

The table shows the results of the investigation of the realtions with delay to conception. Depression and anxiety in women waiting 12 months before next con- 
Depression and anxiety scores during subsequent pregnancy after stillbirth according to time of conception after stillbirth. Confidence intervals for difference between scores for women who had experienced stillbirth and matched controls

\begin{tabular}{|c|c|c|c|c|c|c|}
\hline \multirow[b]{2}{*}{ Variable } & \multicolumn{2}{|c|}{ Conception before 12 months } & \multicolumn{2}{|c|}{ Conception after 12 months } & \multirow{2}{*}{$\begin{array}{l}95 \% \mathrm{Cl} \text { for } \\
\text { difference }\end{array}$} & \multirow[b]{2}{*}{$P$ value } \\
\hline & No of women & Score & No of women & Score & & \\
\hline Depression 3rd trimester* & 31 & 4.0 & 29 & 1.1 & -0.8 to 6.7 & 0.11 \\
\hline State anxiety 3rd trimester & 27 & 12.1 & 28 & 1.9 & 2.0 to 18.5 & 0.02 \\
\hline Trait anxiety 3rd trimester & 27 & 4.6 & 27 & 0.6 & -3.3 to 11.5 & 0.30 \\
\hline Depression 6 weeks ${ }^{\star}$ & 28 & 0.6 & 25 & -0.24 & -2.4 to 4.2 & 0.60 \\
\hline Depression 26 weeks $\dagger$ & 22 & 0.2 & 22 & -1.0 & -3.6 to 6.0 & 0.61 \\
\hline Depression 1 year† & 27 & 3.5 & 26 & -1.7 & 2.0 to 10.4 & 0.01 \\
\hline State anxiety 1 year & 26 & 4.0 & 26 & -5.6 & 1.3 to 17.9 & 0.02 \\
\hline Trait anxiety 1 year & 26 & 3.5 & 26 & -6.8 & 2.0 to 18.6 & 0.02 \\
\hline
\end{tabular}

${ }^{*}$ Edinburgh postnatal depression scale. †Beck depression inventory.

ception were similar to those in the control women at all points, with less trait anxiety $(95 \%$ confidence interval for difference 0.5 to $13.0 ; \mathrm{P}=0.04) 1$ year after birth, while women who next conceived less than 12 months after loss had more symptoms than their control women at all points. Differences between those waiting 12 months before conception and those who conceived sooner were significant in the third trimester for state anxiety $(\mathrm{P}=0.02)$. At 1 year there were significant differences in state and trait anxiety (both $\mathrm{P}=0.02)$ and depression $(\mathrm{P}=0.01$; table).

With dichotomous scoring of Beck's depression inventory at 1 year there was a trend for more above criterion scores for depression between those conceiving before 12 months and those conceiving after 12 months $(28 \%$ v $11 \% ; \mathrm{P}=0.18)$. This compares with only $8 \%$ in the control group scoring above criterion at 1 year (see above). There was a trend for more above criterion scores for depression at any time post partum between those conceiving before 12 months and those conceiving after 12 months $(32 \% v 13 \% ; \mathrm{P}=0.06)$.

Differences between groups remained with a cut off of 15 months to conception but disappeared with cut offs of 9 or 18 months.

Antenatal scores as a predictor of later depression Scores on the Edinburgh postnatal depression scale during the third trimester strongly predicted 12 month follow up scores on Beck's depression inventory (Spearman $r=0.37 ; 0.22$ to $0.54 ; \mathrm{P}<0.0005 ; \mathrm{n}=109$ ). Correlation was notably higher in subjects $(r=0.5 ; 0.27$ to $0.68 ; \mathrm{P}<0.0005 ; \mathrm{n}=55)$ than controls $(r=0.23$; -0.08 to $0.47 ; \mathrm{P}=0.10 ; \mathrm{n}=53$ ).

\section{Discussion}

We have shown that women whose previous pregnancy ended in stillbirth have significantly higher levels of depression and state anxiety in the third trimester of the next pregnancy than control women, are no different from controls at 6 and 26 weeks post partum, and show a trend towards greater depression 1 year after the next birth. When we included time since loss in the analysis, the psychopathology was found to be among women who conceived in the 12 months after stillbirth whereas women who had a gap of 12 or more months between loss and conception were not different from their controls at any point.

One explanation is that women may need a year to recover at least partially from the bereavement. Women who lost a child less than 18 months earlier (that is, before the third trimester assessment) are understandably still grieving. Although this explains the higher symptoms in the third trimester, however, it does not entirely explain the pattern of recovery after the next birth and later increase in depression and anxiety. In the women who conceived after a longer time since loss, those who were 18 or more months after loss when assessed in the third trimester were no more depressed or anxious than control women; yet in women who conceived quickly, 12 months after the birth-that is, 23 to 32 months after loss-they were more depressed and anxious than controls. This gives some support to Lewis's contention that pregnancy interferes with the normal process of mourning. The findings of this study suggest that depression and anxiety may be prolonged for some women who become pregnant within 12 months after stillbirth and at a time when they would most wish to be well.

An alternative explanation is that choosing to become pregnant relatively soon after the loss is partly determined by personality. The lower trait anxiety among slower to conceive women 1 year after the next birth offers some support for this view. It suggests either that those who do not conceive quickly are less anxious than those who conceive sooner or that the longer process of mourning has left mothers who wait less anxious than before.

Women mentioned various determinants in their timing of the next pregnancy, including the longing of one or both parents to replace the lost child as a way of reducing distress, advice from relatives or health professionals, maternal age, and other social factors. Some mothers did not get pregnant soon after loss because the first pregnancy had been unwanted or because they no longer had a partner or because they thought that they needed time to recover physically and psychologically from the trauma. Few current pregnancies were unplanned, and those few were failure to use contraception rather than failure of contraception.

\section{Advice to parents}

The decision to have another child after late loss in pregnancy is a personal one, but parents often seek professional advice about timing the next pregnancy, although they resent being told categorically when to go ahead. ${ }^{21}$ We have shown that conception within a year after stillbirth was associated with higher levels of depression and anxiety in pregnancy compared with conception later. Most women recovered after the birth, but those who had conceived more quickly were again at higher risk of depression and anxiety when 
born subsequent to late pregnancy loss: case-control study. Prenat Diag 1997; 17:843-51

9 Wadwa PD, Sandman CA, Porto M, Dunkel-Schetter C, Garite U. The association between prenatal stress and infant birth weight and gestational age at birth: a prospective investigation. Am J Obstet Gynecol 1993;69:858-65.

- Women whose previous pregnancy ended in stillbirth had significantly higher levels of depression and state anxiety during their subsequent pregnancy than matched controls

- Those who had conceived over 12 months after stillbirth were, however, similar to controls at all points and had lower trait anxiety a year after the next birth

- Women who had conceived within 12 months after loss had a significantly higher risk of high state anxiety during the next pregnancy and of depression and both state and trait anxiety 12 months post partum than women with longer time since loss

- Women may need a year to mourn the lost child before beginning another pregnancy or women who chose to conceive sooner may be intrinsically more vulnerable to depression and anxiety

- Parents have various and individual reasons for timing the next pregnancy, but there may be advantage in waiting 12 months before conception

their baby was a year old. Personality could be the common determinant both of conceiving sooner and of higher psychopathology, but the need for adequate time to grieve is a common sense explanation and likely contributor.

Parents naturally want to know what there is to know about relative risks and may want to have the information and decide for themselves. It should also be noted that most women who went ahead quickly with another pregnancy did not experience high levels of anxiety and depression, and for some parents other considerations may outweigh the possible higher risk of psychological symptoms.

We thank Liz Hopper, Gill McGauley, and David Fainman, who contributed to the data collection, the obstetricians and midwives at St George's, St Helier's, and Kingston Hospitals, and particularly the women who allowed us to interview them.

Contributors: PMH initiated the study, participated in data collection, and wrote the paper. PT did most of the data collection, organised the data, and discussed ideas. CDHE carried out the statistical analysis and discussed ideas and interpretation od the findings. PMH is the guarantor.

Funding: South Thames Research and Development, the Simenauer Trust (Institute of Psychoanalysis), and Tommy's Campaign.

Competing interests: None declared.

1 Office for National Statistics. Birth statistics 1996, England and Wales. London: Office for National Statistics, 1998:1. (Series FMI, No 25.)

2 Forrest GC, Standish E, Baum JD. Support after perinatal death: a study of support and counselling after perinatal bereavement. BMJ 1982;285:1475-9

3 Lewis E. Inhibition of mourning by pregnancy: psychopathology and management. $B M J 1979 ; \mathrm{ii}: 27-8$.

4 Rowe J, Clyman R, Green C. Follow-up of families who experience a perinatal death. Pediatrics 1978;62:166-70.

5 Bourne S, Lewis E. Delayed psychological effects of perinatal deaths: the next pregnancy and the next generation. BMJ 1984;289:147-8.

6 Phipps S. The subsequent pregnancy after stillbirth: anticipatory parenthood in the face of uncertainty. Int J Psych Med 1985-6;15:243-64.

7 Theut SK, Pedersen FA, Zaslow MJ, Rabinovich BA. Pregnancy subsequent to perinatal loss: parental anxiety and depression. J Amer Acad Child Adolesc Psychiatry 1988;27:289-92.

8 Hunfeld JAM, Taselaar AKG, Agterberg G, Wladimiroff JW, Passchier J. Trait anxiety, negative emotions and the mothers' adaptation to an infant
10 Copper RL, Goldenberg RL, Das A, Elder N, Swain M, Norman G, et al. The preterm prediction study: maternal stress is associated with spontaneous preterm birth at less than 35 weeks gestation. Am J Obstet Gynecol 1996;175:1286-92.

11 Lou HC, Hansen D, Nordentoft, Pryds O, Jensen F, Nim J, et al. Prenatal stressors of human life affect fetal brain development. Dev Med Child Neurol 1994;36:826-32.

12 Teixeira JMA, Fisk NM, Glover V. Association between maternal anxiety in pregnancy and increased uterine artery resistance index: cohort based study. BMJ (in press).

13 Gitau R, Cameron A, Fisk NM, Glover V. Fetal exposure to maternal cortisol. Lancet 1998;352:707-8.

14 Zuckerman B, Amaro, A, Bauchner H, Cabral H. Depressive symptoms during pregnancy: relationship to poor health behaviors. Am J Obstet Gynecol 1989;160:1107-11.

15 Murray L. The impact of postnatal depression on infant development. J Child Psychol Psychiatry 1992;33:543-62.

16 Sroufe LA. Infant-caregiver attachment and patterns of adaptation in $\mathrm{M}$, ed. Minnesota symposium in child psychology. Vol 6. Hillsdale, NJ: Erlbaum, 1983:41-81.

17 Cox J, Holden JM, Sagovsky R. Detection of postnatal depression: development of the Edinburgh postnatal depression scale. Br J Psych 1987;150:782-6.

18 Murray D, Cox J. Screening for depression during pregnancy with the Edinburgh depression scale (EPDS). J Repro Infant Psychol 1990;8:99-107.

19 Beck AT, Ward CH, Mendelson M, Mock J, Erbaugh J. An inventory for measuring depression. Arch Gen Psychiatry 1961;4:561-9.

20 Spielberger CD, Gorsuch RL, Lushene R. Test for the state-trait anxiety

21 Davis DL, Stewart M, Harmon RJ. Postponing pregnancy after perinatal death: perspectives on doctor advice. J Amer Acad Child Adolesc Psychiatry 1989;28:481-7.

(Accepted 26 March 1999)

\section{Corrections and clarifications}

Prescribing antibiotics for sore throats

In this letter by Morten Lindbaek (24 April, pp

1138-9) the author's name was misspelt as Morten Lindbæk.

Dutch system of peer review is different and effective At the end of the first paragraph of this letter by Peter Bourdillon (24 April, p 1143) the reference Ear-Nose-Throat Society, March 1998) should have been: van der Baan S. Peer review: experiences of the Dutch Ear-Nose-Throat Society. In: CMEmaking sure it works. London: Department of Health, Academy of Medical Royal Colleges, 1998:62-4. (www.open.gov.uk/doh/meconf.htm).

Sexual health of teenagers in England and Wales:

Two errors occurred in this article by Angus Nicoll and colleagues (15 May, pp 1321-2). The first sentence of the second paragraph of the subjects, methods, and results section should have started as: "In 1995 [not 1996] there were...." In the table (p 1321) the data on conceptions leading to terminations of pregnancy or maternities were for women under 20 years old, not for those aged 16-19 (for terminations) or $<16-19$ (for maternities). Rates for women aged under 20 were based on the population of women aged 15-19. Routine data on conceptions in England and Wales are published in the Office for National Statistics' Birth Statistics, series FMI and available from the Stationery Office, London.

Obituaries

In the obituary of Henry Gordon Smylie (17 April, p 1080) the edited text referred to Dr Smylie as Henry; he was always known as Gordon. In the obituary of William Ian Leslie Fraser (1 May, p 1217) the edited text referred to Dr Fraser as William; he was always known as Ian. pre-school: the roots of maladaptation and competence. In: Perlmutter inventory. Palo Alto: Consulting Psychologists Press, 1970. given ( $\mathrm{S}$ van der Baan, conference of the Dutch analysis of national data 